OPEN ACCESS

Edited by:

Wolfgang R. Streit, University of Hamburg, Germany

Reviewed by: Dave Siak-Wei Ow, Bioprocessing Technology Institute, A*STAR, Singapore Nicole Frankenberg-Dinkel, Kaiserslautern University of

Technology, Germany

*Correspondence: Amanda G. Oglesby-Sherrouse aoglesby@rx.umaryland.edu

Specialty section: This article was submitted to Systems Microbiology,

a section of the journal Frontiers in Microbiology

Received: 02 June 2016 Accepted: 14 July 2016 Published: 27 July 2016

Citation: Nguyen AT, Jones JW, Cámara M, Williams $P$, Kane MA and Oglesby-Sherrouse AG (2016) Cystic Fibrosis Isolates of Pseudomonas aeruginosa Retain Iron-Regulated Antimicrobial Activity against Staphylococcus aureus through the Action of Multiple Alkylquinolones. Front. Microbiol. 7:1171.

doi: 10.3389/fmicb.2016.01171

\section{Cystic Fibrosis Isolates of} Pseudomonas aeruginosa Retain Iron-Regulated Antimicrobial Activity against Staphylococcus aureus through the Action of Multiple Alkylquinolones

\author{
Angela T. Nguyen ${ }^{1}$, Jace W. Jones ${ }^{1}$, Miguel Cámara ${ }^{2}$, Paul Williams ${ }^{2}$, Maureen A. Kane ${ }^{1}$ \\ and Amanda G. Oglesby-Sherrouse ${ }^{1,3 *}$
}

\begin{abstract}
${ }^{1}$ Department of Pharmaceutical Sciences, School of Pharmacy, University of Maryland, Baltimore, MD, USA, ${ }^{2}$ Centre for Biomolecular Sciences, School of Life Sciences, University of Nottingham, Nottingham, UK, ${ }^{3}$ Department of Microbiology and Immunology, School of Medicine, University of Maryland, Baltimore, MD, USA
\end{abstract}

Cystic fibrosis (CF) is a hereditary disease that predisposes individuals to pulmonary dysfunction and chronic infections. Early infection of the CF lung with Staphylococcus aureus is common, while Pseudomonas aeruginosa becomes dominant as disease progresses. Emergence of $P$. aeruginosa likely depends on the action of multiple 2-alkyl-4-(1H)-quinolones (AQ) secreted by this organism. We recently showed that antimicrobial activity against $S$. aureus is enhanced by iron depletion and is dependent upon multiple $A Q$ metabolites. Two of these AQs, the Pseudomonas quinolone signal [PQS; 2-heptyl-3-hydroxy-4(1H)-quinolone] and 2-heptyl-4-hydroxyquinoline $(\mathrm{HHQ})$, are quorum sensing molecules that activate the expression of multiple microbicidal factors. Here we show for the first time that $\mathrm{HHQ}$ also exhibits innate antimicrobial activity against $S$. aureus. We further show that iron depletion potentiates the antistaphylococcal activity of $\mathrm{HHQ}$, as well as 2-heptyl-4-hydroxyquinoline- $N$-oxide (HQNO), another $\mathrm{AQ}$ that functions as a cytochrome B inhibitor. Notably, we found that deletion of the genes for the terminal biosynthetic steps for either PQS or HQNO results in overproduction of the $\mathrm{HHQ}$ intermediate, likely maintaining the ability of these mutants to mediate antimicrobial activity. Compensatory increases in $\mathrm{HHQ}$ were also observed in PQS-deficient CF isolates, which also retained the ability to mediate iron-regulated antimicrobial activity against $S$. aureus. These studies demonstrate that iron-regulated antimicrobial activity of $P$. aeruginosa against $S$. aureus is due to the cumulative effects of multiple $A Q$ metabolites, both the production and activity of which are modulated by environmental iron levels.

Keywords: Pseudomonas aeruginosa, iron regulation, AQs, Staphylococcus aureus, quorum sensing 


\section{INTRODUCTION}

CF is a hereditary disease that predisposes patients to pulmonary dysfunction and chronic infection by multiple microorganisms, including Staphylococcus aureus and Pseudomonas aeruginosa. While $S$. aureus is one of the dominant pathogens during early CF lung infection, $P$. aeruginosa eventually becomes the predominant pathogenic resident in the CF lung and persists for decades as a chronic infection (Hoiby et al., 2010; Cystic Fibrosis Foundation, 2014). The factors that contribute to this shift are not well understood, but isolation of $P$. aeruginosa from the lungs of CF patients is correlated with increased exacerbation and a decline in lung function (Rabin et al., 2004; Bhatt, 2013). Thus, understanding the factors that contribute to long term survival of $P$. aeruginosa in the CF lung is essential to understanding disease progression in these patients.

2-alkyl-4-(1H)-quinolones (AQs) produced by $P$. aeruginosa are hypothesized to contribute to the eventual dominance of this pathogen in the CF lung (Mashburn et al., 2005). AQ production requires the co-enzyme ligase PqsA, which converts a cellular metabolite, anthranilate, to its active form, anthraniloyl CoA (Figure 1; Coleman et al., 2008). This in conjunction with fatty acids and the PqsBCD enzyme complex results in the production of over 55 distinct AQs (Coleman et al., 2008; Dulcey et al., 2013; Drees et al., 2016). AQs exhibit a variety of functions including cell signaling, redox activity, and antimicrobial activity (Deziel et al., 2004; Diggle et al., 2007). Perhaps the best studied AQs are 2-heptyl-4-hydroxyquinoline (HHQ; Wratten et al., 1977) and the Pseudomonas quinolone signal [PQS; 2-heptyl-3-hydroxy4(1H)-quinolone; Calfee et al., 2001], which function as quorum signaling molecules and induce the expression of virulenceassociated genes. Included in the PQS/HHQ regulon are genes required for the production of exoenzymes, lectins, siderophores (pyochelin and pyoverdine), and phenazines (Dietrich et al., 2006; Diggle et al., 2007; Rampioni et al., 2010). Phenazines are redox active metabolites produced by $P$. aeruginosa that generate reactive oxygen species resulting in the lysis of both human and microbial cells (Price-Whelan et al., 2006). Exoenzymes such as elastases contribute to the lysis of $S$. aureus by cleaving the peptidoglycan pentaglycine interpeptides of the cell wall (Kessler et al., 1993, 1997). The PqsABCD biosynthetic pathway in conjunction with the mono-oxygenase PqsL (Heeb et al., 2011) can also generate AQ N-oxides (AQNOs) such as 2-heptyl-4hydroxyquinoline- $N$-oxide (HQNO), which suppresses growth of gram-positive bacteria such as S. aureus (Figure 1; Van Ark and Berden, 1977; Machan et al., 1992; Smirnova et al., 1995; Rothery and Weiner, 1996; Hoffman et al., 2006).

Another major factor in $P$. aeruginosa's ability to survive in the complex microbial environment of the CF lung is the acquisition of essential nutrients, including iron. $P$. aeruginosa requires iron for growth and virulence (Meyer et al., 1996; Takase et al., 2000; Xiong et al., 2000; Nadal Jimenez et al., 2010), yet this element is sequestered during infection by multiple host proteins (Otto et al., 1992; Nairz et al., 2010). To obtain iron from the host, $P$. aeruginosa expresses multiple high affinity iron uptake systems, which have been studied extensively over the past three decades (Cornelis, 2010; Cornelis and Dingemans, 2013; Konings et al., 2013). Previously, it was reported that P. aeruginosa could also lyse and acquire iron from $S$. aureus through the secretion of AQs (Mashburn et al., 2005), the production of which is stimulated by peptidoglycan released from $S$. aureus upon cell lysis (Korgaonkar et al., 2013). A recent report also showed that the acquisition of iron by siderophores is required for efficient killing of $S$. aureus by HQNO in both planktonic and biofilm culture environments (Filkins et al., 2015). Coinciding with this report, we showed that production of a PQS congener with a longer alkyl chain is enhanced by iron depletion, correlating with increased antimicrobial activity against $S$. aureus in irondepleted environments (Nguyen et al., 2015). Combined, these studies demonstrate a complex relationship between iron and AQ-mediated interactions of $P$. aeruginosa and S. aureus.

In the current work, we sought to determine how individual AQs produced by $P$. aeruginosa contribute to iron-regulated antimicrobial activity against $S$. aureus. We show that, in addition to its roles as a quorum sensing molecule, HHQ exhibits innate antimicrobial activity against $S$. aureus. We further show that iron depletion potentiates the innate antimicrobial activity of HHQ and HQNO against S. aureus. Despite significant changes in AQ metabolites incurred by clonal, longitudinal CF isolates, we demonstrate that iron-regulated antimicrobial activity against $S$. aureus is largely retained by these isolates. We postulate that conservation of this phenomenon is due to the cumulative effects of multiple AQ metabolites, both the production and activity of which are modulated by environmental iron levels.

\section{MATERIALS AND METHODS}

\section{Strains and Growth Media}

$P$. aeruginosa and $S$. aureus strains used in this study are shown in Table 1. CF isolates of $P$. aeruginosa were originally obtained from Dr. David Speert and previously described by our lab (Nguyen et al., 2014). Brain heart infusion (BHI) was used for routine culture of $S$. aureus and $P$. aeruginosa. Dialyzed trypticase soy broth (DTSB) for iron studies was prepared as previously described for iron-depleted medium (Oglesby-Sherrouse et al., 2014). Cultures were supplemented with $100 \mu \mathrm{M} \mathrm{FeCl}$ as indicated.

\section{Antimicrobial Assays}

To prepare extracts for antimicrobial assays, $P$. aeruginosa strains were grown in DTSB medium supplemented with or without 100 $\mu \mathrm{M} \mathrm{FeCl} 3$ for $18 \mathrm{~h}$ at $37^{\circ} \mathrm{C}$. $\mathrm{OD}_{600}$ of cultures was measured and cultures were centrifuged for $5 \mathrm{~min}$ at 14,000 RPM $(\sim 16,000$ $\times \mathrm{g})$ in a tabletop centrifuge. Supernatant volume collected for extraction was normalized to the lowest culture OD in each biological replicate. Supernatants were extracted as previously described (Collier et al., 2002) and $1 \mathrm{ml}$ was evaporated to dryness. Dried extracts were resuspended in $20 \mu \mathrm{L}$ of $100 \%$ ethyl alcohol. For antimicrobial assays, the methicillin-resistant $S$. aureus strain M2 was grown for $18 \mathrm{~h}$ in $\mathrm{BHI}$ medium at $37^{\circ} \mathrm{C}$. Growth was measured by $\mathrm{OD}_{600}$, then diluted to an $\mathrm{OD}$ 0.05 in DTSB medium supplemented with or without $100 \mu \mathrm{M}$ $\mathrm{FeCl}_{3} .1 \mu \mathrm{L}$ of $P$. aeruginosa extracts was added to $200 \mu \mathrm{L}$ of diluted S. aureus culture. $1 \mu \mathrm{L} 100 \%$ ethyl alcohol was used as a 
A

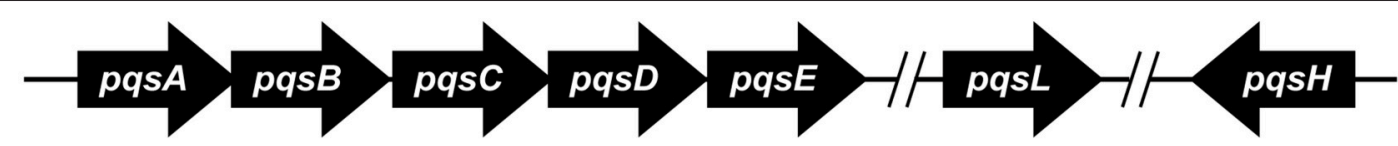

B<smiles>[Y][AsH]S(=O)(=O)c1ccccc1N</smiles>

Anthranilic acid<smiles>CC(=O)SC(=O)c1ccccc1N</smiles>

PqsBCD Anthraniloyl-CoA<smiles>CCCC</smiles>

C

\begin{tabular}{|c|c|c|}
\hline Abbreviation & Nomenclature & Description \\
\hline$A Q$ & 2-alky|-4-(1H)-quinolone & alkyl quinolones \\
\hline HHQ & 2-hepty-4-hydroxyquinoline & C7-alkyl chain \\
\hline NHQ & 2-nonyl-4-hydroxyquinoline & c9-alkyl chain \\
\hline PQS & 2-heptyl-3-hydroxy-4(1H)-quinolone & C7-alkyl chain; Pseudomonas quinolone signal \\
\hline C9-PQS & 2-nonyl-3-hydroxy-4(1H)-quinolone & C9-alkyl chain; Pseudomonas quinolone signal \\
\hline AQNO & 2-alky-4-hydroxyquinoline $N$-oxide & alkyl quinoline $\mathrm{N}$-oxides \\
\hline HQNO & 2-heptyl-4-hydroxyquinoline $\mathrm{N}$-oxide & C7-alkyl chain $\mathrm{N}$-oxide \\
\hline NQNO & 2-nonyl-4-hydroxyquinoline $N$-oxide & C9-alkyl chain $\mathrm{N}$-oxide \\
\hline
\end{tabular}<smiles>O=[N+]([O-])O[N+](=O)[O-]</smiles><smiles>[R]c1cc(=O)c2ccccc2[nH]1</smiles><smiles>C1CCCCC1</smiles>

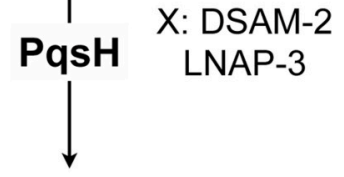

FIGURE 1 | AQ biosynthesis in P. aeruginosa. (A,B) Genetics of AQ biosynthesis. Anthranilic acid is converted to anthraniloyl-CoA by PqsA. The genes encoding PqsBCD, which contributes to the production of $\mathrm{HHQ}$ and congeners, are located in an operon with PqsA. The Pqs $\mathrm{L}$ and PqsH enzymes, which mediate synthesis of HQNO and PQS respectively, and related congeners are encoded at distal sites of the chromosome. Each of these AQ core structures varies with respect to the length and saturation of their alkyl chain (-R). Specific CF isolates of $P$. aeruginosa (JSRI-2, DSAM-2, and LNAP-3) are hypothesized to defective at distinct steps in the $A Q$ metabolic pathway as indicated. (C) $A Q$ abbreviations used in this study.

TABLE 1 | Strains used in this study.

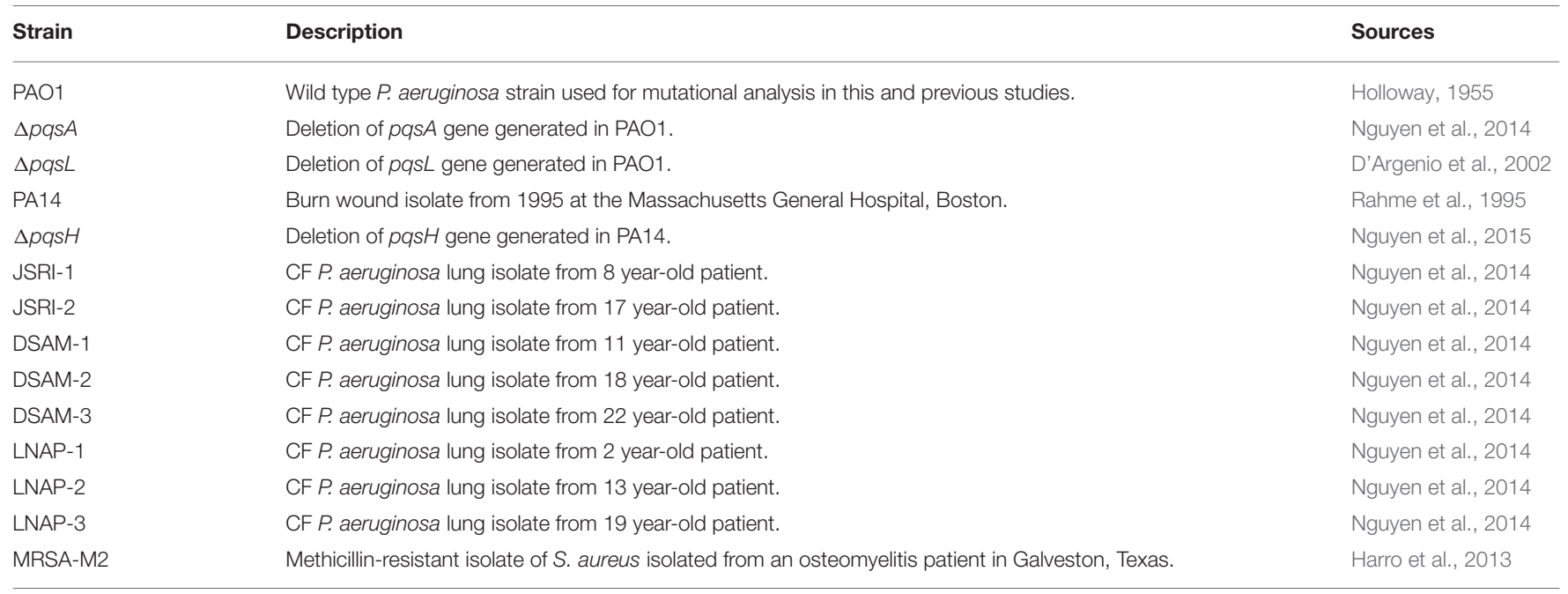

solvent control. Cultures were incubated for $18 \mathrm{~h}$ with shaking at $37^{\circ} \mathrm{C}$ in a 96 -well plate, and $S$. aureus cell density $\left(\mathrm{OD}_{630}\right)$ was measured spectroscopically in a BioTek ${ }^{\circledR}$ Synergy ${ }^{\mathrm{TM}}$ HT plate reader.

\section{Mass Spectrometric Quantification of AQs}

Strains were grown for $18 \mathrm{~h}$ in DTSB supplemented with or without $100 \mu \mathrm{M} \mathrm{FeCl} 3$ as indicated. Cells were spun down and supernatants harvested for AQ extraction as previously 
described by Collier et al. (2002). Nalidixic acid and deuterated $\mathrm{N}$-dodecanoyl-L-homoserine lactone $\left(\mathrm{C}_{12}\right.$-HSL) were used as internal standards. LC-MS/MS analysis was performed as previously described (Ortori et al., 2011; Nguyen et al., 2015).

\section{Transwell Co-culture Assay}

To quantify antimicrobial activity against $S$. aureus, a liquid co-culture system using transwell cell culture inserts (Corning Costar $^{\circledR}$, NY, USA) was performed as previously described (Nguyen et al., 2015). Briefly, P. aeruginosa and S. aureus strains were grown overnight in DTSB for $18 \mathrm{~h}$ at $37^{\circ} \mathrm{C}$. S. aureus cultures were diluted to an $\mathrm{OD}_{600}$ of 0.05 in DTSB supplemented with or without $100 \mu \mathrm{M} \mathrm{FeCl}_{3}$, and $600 \mu \mathrm{L}$ of the resulting cell suspension was inoculated into the bottom of the transwell plate. A transwell insert with a $0.4 \mu \mathrm{m}$ membrane was then placed onto the plate, and $100 \mu \mathrm{L}$ of $P$. aeruginosa cultures, diluted to an $\mathrm{OD}_{600}$ of 0.05 , were inoculated on top of the membrane. The transwell plates were incubated at $37^{\circ} \mathrm{C}$ for $18 \mathrm{~h}$ under static growth conditions, and $S$. aureus cell density $\left(\mathrm{OD}_{630}\right)$ was measured spectroscopically in a BioTek ${ }^{\circledR}$ Synergy $^{\mathrm{TM}}$ HT plate reader.

\section{Real Time PCR Analysis}

Real time PCR (qPCR) analysis of $P$. aeruginosa and $S$. aureus gene expression in broth cultures was carried out as previously described (Oglesby et al., 2008; Nguyen et al., 2014; Reinhart et al., 2015), using the Applied Biosystems StepOne Plus Real Time PCR System (Life Technologies). Primers and probes used in this study are listed in Table $S 1$. For $S$. aureus cell lysis: cell pellets were resuspended in $100 \mu \mathrm{L}$ of $2.5 \mu \mathrm{g} / \mu \mathrm{L}$ lysozyme and $0.25 \mu \mathrm{g} / \mu \mathrm{L}$ lysostaphin in TE and incubated for $45 \mathrm{~min}$ at $37^{\circ} \mathrm{C}$. Relative amounts of cDNA were determined by the $\Delta \Delta \mathrm{C}_{\mathrm{T}}$ method, and expression was normalized to oprF cDNA for P. aeruginosa or $r p o B$ cDNA for $S$. aureus detected in each sample.

\section{Thin Layer Chromatography (TLC)}

Bacteria were grown in DTSB for $18 \mathrm{~h}$ at $37^{\circ} \mathrm{C}$, with or without $100 \mu \mathrm{M} \mathrm{FeCl} 3$ supplementation as indicated. Each culture was harvested and extracted with acidified ethyl acetate as described by Collier et al. (2002). One half of the resulting organic extract was transferred to a clean tube and evaporated to dryness. Samples were resuspended in 1:1 acidified ethyl acetate:acetonitrile and analyzed by thin-layer chromatography (TLC) with a synthetic PQS standard (Pesci et al., 1999).

\section{RESULTS}

\section{Antimicrobial Activity of Alkylquinoline- $N$-oxides (AQNOs) is Dependent on S. aureus Growth Environment}

Previous analysis of a panel of pqs mutants demonstrated that antimicrobial activity of $P$. aeruginosa against $S$. aureus is dependent upon multiple AQs, including HQNO (Filkins et al., 2015; Nguyen et al., 2015). HQNO possesses innate antimicrobial activity against $S$. aureus due to its ability to bind to and inhibit the activity of cytochrome b (Lightbown and Jackson, 1956; Machan et al., 1992). Recent studies also demonstrate that both siderophores and AQNOs are required for antimicrobial activity against $S$. aureus during mixed biofilm growth (Filkins et al., 2015), indicating that iron may play a role in AQNO-mediated antimicrobial activity. We therefore sought to determine how iron supplementation affected the antimicrobial activity of AQNOs against $S$. aureus. Since $P$. aeruginosa produces several congeners of AQNOs, with varying alkyl chain lengths and levels of saturation, we chose to first analyze extracts of $P$. aeruginosa culture supernatants instead of assaying the effects of individual AQNO metabolites. AQs were extracted from wild type PAO1, the $\triangle p q s A$ mutant, which lacks production of all AQs, and the $\Delta p q s L$ mutant, which is specifically defective for AQNO production (Figure 1). S. aureus was then cultured in high or low iron media in the presence of each of these extracts. As expected, wild type PAO1 extracts substantially reduced S. aureus growth (by 50\%) as compared to the solvent control (Figure 2A). This effect was eliminated when $S$. aureus cultures were subjected to either the $\triangle p q s A$ or $\triangle p q s L$ extracts (Figure 2A), demonstrating the specific role of AQNOs in inhibiting $S$. aureus growth in this assay. Notably, iron supplementation of $P$. aeruginosa cultures had no impact on the ability of the resulting extracts to inhibit $S$. aureus growth (Figure 2A). In contrast, iron depletion of $S$. aureus cultures significantly enhanced the antimicrobial activity of both the high and low iron PAO1 extracts (Figure 2A). This was not due to effects of the Pseudomonas growth medium, as extracted high or low iron DTSB media did not significantly affect $S$. aureus growth (Supplementary Figure S1). These data indicate that while iron does not affect on the production of AQNOs by P. aeruginosa, it does impact the susceptibility of $S$. aureus cultures to these metabolites.

To directly determine if AQNO production is regulated by iron, we quantified AQNO levels in supernatants of P. aeruginosa cultures grown in low or high iron media using liquid chromatography tandem mass spectrometry (LC-MS/MS). For this analysis we selected HQNO, an AQNO congener with a C7 (heptyl) alkyl chain, and NQNO, an AQNO congener with a C9 (nonyl) alkyl chain. Strikingly, our results show that the impact of iron on AQNO production is dependent upon alkyl chain length: production of HQNO is repressed by iron depletion, while that of NQNO is induced by iron depletion (Figures 2B,C). These data further support the idea that iron depletion does not induce overall AQNO production by $P$. aeruginosa, and that antimicrobial activity of AQNOs is instead dependent upon iron depletion of $S$. aureus cultures. To directly test this idea, we subjected $S$. aureus low and high iron cultures to HQNO and NQNO synthesized as described previously (Ortori et al., 2011). While HQNO and NQNO suppressed growth of $S$. aureus when cultured in either high iron medium, iron depletion significantly enhanced the antimicrobial activity of these metabolites (Figure 3A). As expected, decreases in cell density correlated closely with changes in cellular respiration as determined by staining cultures with triphenyltetrazolium chloride (TTC), indicating that the effects of AQNOs are due to inhibition of cytochrome activity vs. loss of cell viability (Table S2). Combined, these results show that iron starvation 
A

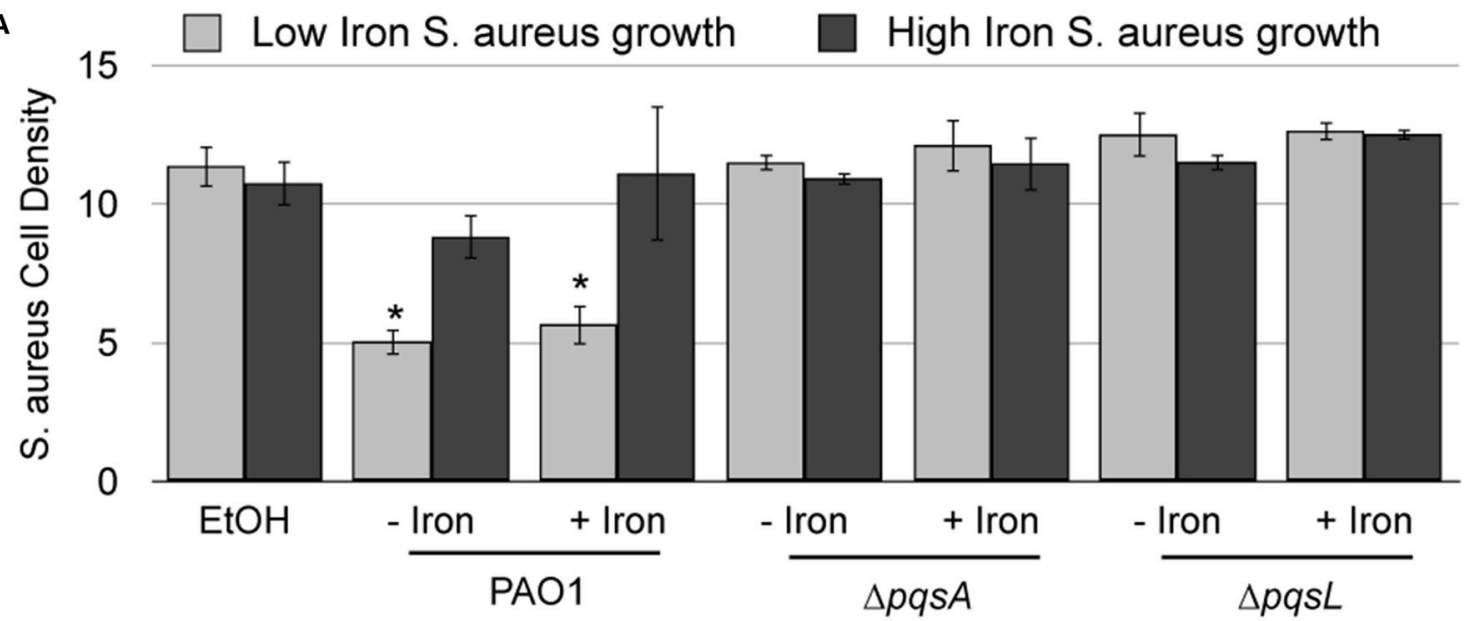

B

HQNO

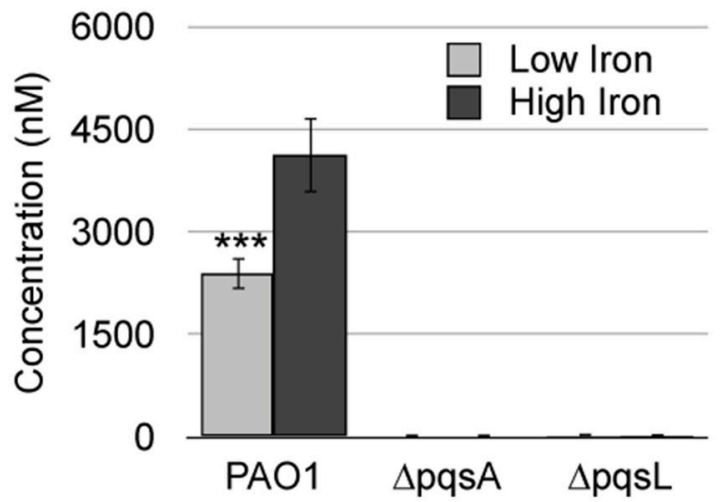

C

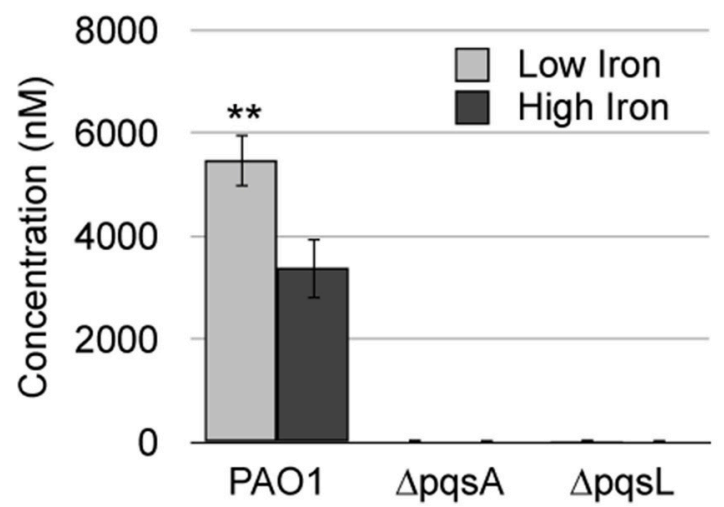

FIGURE 2 | AQNO-dependent antimicrobial activity against S. aureus is dependent upon iron depletion. (A) S. aureus was grown overnight in DTSB supplemented with or without $100 \mu \mathrm{M} \mathrm{FeCl}_{3}$ and with the indicated $P$. aeruginosa $\mathrm{AQ}$ extracts, prepared as described in the Section Materials and Methods. (B) Culture supernatant extracts from the indicated $P$. aeruginosa strains were prepared and analyzed by LC-MS/MS as described in the Section Materials and Methods. Error bars indicate standard deviation of three $\mathbf{( A )}$ or five $\mathbf{( B )}$ biological replicates. Asterisks $\left({ }^{*}\right)$ indicate the following $p$-values as determined by two-tailed Student's t-test: ${ }^{\star} p<0.05,{ }^{* *} p<0.005,{ }^{\star * *} p<0.0005$ when comparing low iron to high iron.

sensitizes $S$. aureus cultures to the antimicrobial activity of AQNOs.

\section{HHQ Displays Innate Antimicrobial Activity against $S$. aureus}

We next sought to determine how PQS and HHQ contribute to iron-regulated antimicrobial activity against $S$. aureus. PQS and HHQ both function as quorum sensing molecules, activating the expression of genes that contribute to $S$. aureus growth suppression (Deziel et al., 2004; Diggle et al., 2007). PQS is additionally able to chelate iron (Bredenbruch et al., 2006; Diggle et al., 2007), which could also contribute to growth suppression of $S$. aureus. Our previous work showed that $p q s H$, which is responsible for the conversion of HHQ to PQS (Figure 1), is not required for $S$. aureus growth suppression during co-culture (Nguyen et al., 2015). Deletion of $p q s H$ also had no effect on the antimicrobial activity of culture supernatant extracts (Figure 4A). Combined, these results indicate the iron-chelation activity of PQS is dispensable for $S$. aureus growth suppression. Alternatively, it is possible that deletion of $p q s H$ alters the production of AQ metabolites, resulting in enhanced antimicrobial activity by other mechanisms. We therefore used LC-MS/MS to quantify production of HHQ and $\mathrm{HQNO}$, and related congeners, in the $\Delta p q s H$ mutant. While no significant changes in either HQNO or NQNO were observed (Supplementary Figures S2C,D), these results showed more than a 10 -fold increase in HHQ and NHQ production in the $\Delta p q s H$ mutant as compared to the wild type strain in both high and low iron conditions (Figures $4 \mathrm{~B}, \mathrm{C}$ ). It is therefore possible that increased production of HHQ suppresses defects in antimicrobial activity that are specific to loss of PQS.

We next determined if PQS, due to its ability to chelate iron, exhibits innate antistaphylococcal activity by subjecting $S$. aureus low and high iron cultures to PQS and related congeners (C9-PQS and C1-PQS). Interestingly, only C9-PQS caused a small but significant decrease in $S$. aureus culture 


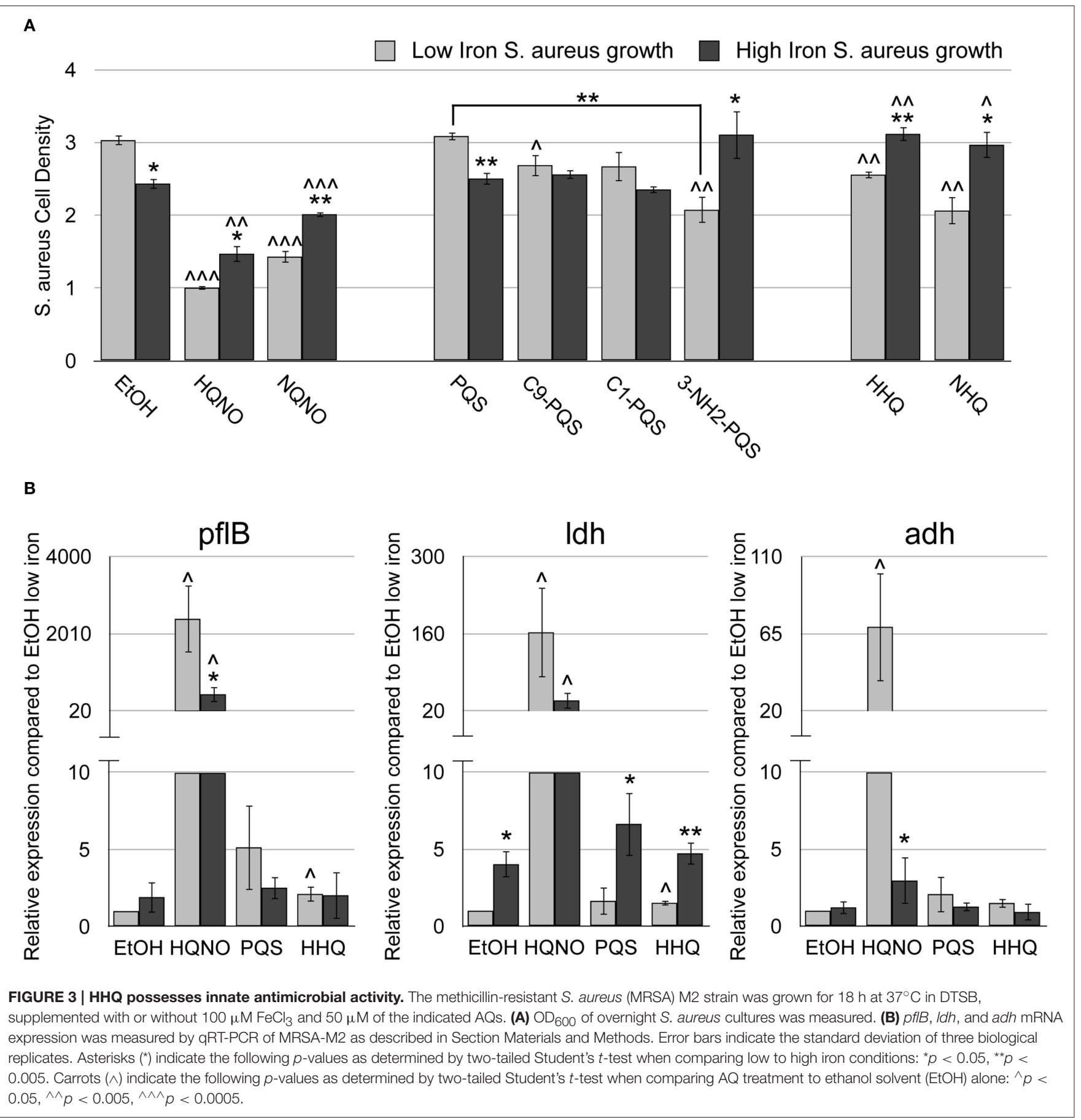

density as compared the ethanol control $(p<0.05)$, and this effect was only observed in low iron (Figure 3A). To determine if this activity was due to iron chelation, we also treated $S$. aureus cultures with $\mathrm{NHQ}$ and $\mathrm{HHQ}$, as well as a PQS mimic, 3- $\mathrm{NH}_{2}-\mathrm{PQS}$. The replacement of an amino group for the hydroxyl group on the $3^{\prime}$ position of the quinolone allows for binding and activation of PqsR, but not iron chelation ability (Ilangovan et al., 2013). Surprisingly, 3- $\mathrm{NH}_{2}-\mathrm{PQS}, \mathrm{HHQ}$, and NHQ were all able to suppress growth of $S$. aureus in low iron (Figure 3A), indicating that the $3^{\prime}$ hydroxyl group of PQS inhibits the growth suppressive activity of these AQs. As observed for HQNO, the reduction in $S$. aureus culture density upon HHQ supplementation correlated with decrease in TTC staining, demonstrating a similar mechanism of growth inhibition (Table S2). Our analysis further shows that addition of either PQS or HHQ has an additive effect on $S$. aureus growth when provided in combination with HQNO (Supplementary Figure S3). The combination of all three AQs did not significantly alter 
A
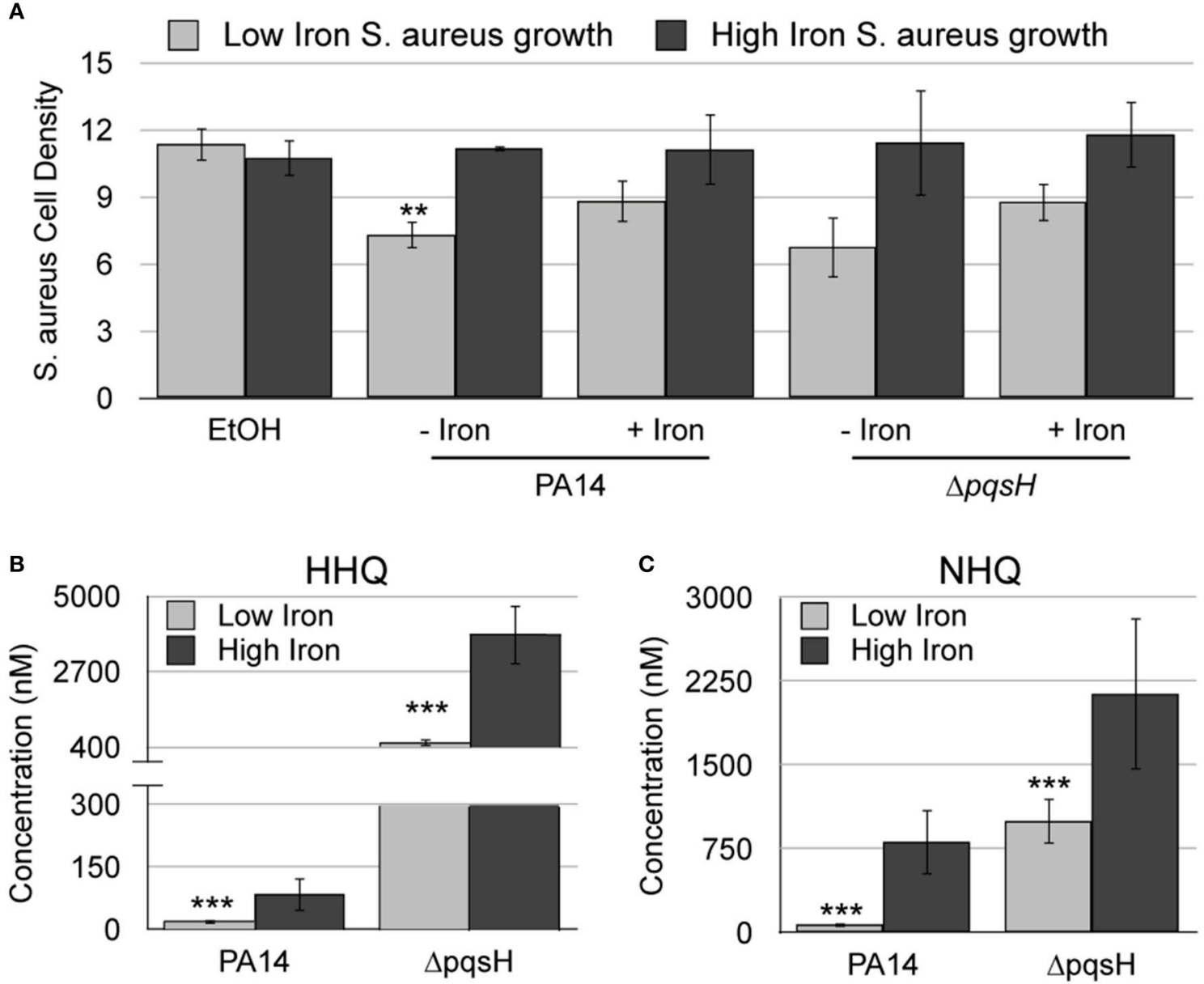

FIGURE 4 | $\mathbf{H H Q}$ is overproduced by the $\mathbf{\Delta} \mathbf{p q s} \boldsymbol{H}$ mutant. (A) S. aureus was grown overnight in DTSB supplemented with or without $100 \mu \mathrm{M}$ FeCl 3 and the indicated $P$. aeruginosa $A Q$ extracts. $\mathrm{OD}_{600}$ of overnight $S$. aureus cultures was measured as described in Section Materials and Methods. (B,C) Culture supernatant extracts from the indicated $P$. aeruginosa strains, grown with or without $100 \mu \mathrm{M} \mathrm{FeCl}_{3}$ were analyzed by LC-MS/MS as described in the Section Materials and Methods. Error bars indicate standard deviation of three (A) and five (B) biological replicates. Asterisks (*) indicate the following $p$-values as determined by two-tailed Student's $t$-test: ${ }^{* \star} p<0.005,{ }^{* \star *} p<0.0005$ when comparing low iron to high iron.

the growth suppression of $S$. aureus compared to HQNO in combination with either PQS or HHQ (Supplementary Figure S3). These data demonstrate a novel role for these signaling molecules in mediating antimicrobial activity against $S$. aureus.

\section{HQNO and HHQ Induce Fermentation Pathways in $S$. aureus}

A recent report by Filkins et al. demonstrated that co-culture with $P$. aeruginosa significantly altered $S$. aureus gene expression in an AQNO-dependent manner (Filkins et al., 2015). The most up-regulated genes were associated with fermentation pathways of $S$. aureus including formate acetyltransferase $(p f l B)$, L-lactate dehydrogenase (ldh), and alcohol dehydrogenase ( $a d h)$. To determine whether synthesized AQNOs were able to induce these same genes, we performed real time PCR on $S$. aureus cultures grown with HQNO in DTSB supplemented with or without iron. HQNO induced $p f l B, l d h$, and $a d h$ gene expression 100-1000 fold in low iron compared to the ethanol control, similar to that observed by Filkins et al. (Figure 3B). Strikingly, HQNO's ability to induce $p f B$, $l d h$, and $a d h$ expression in $S$. aureus was reduced in iron-replete conditions (Figure 3B). Thus, iron levels significantly impact on the ability of HQNO to induce fermentative metabolism genes in S. aureus.

The studies by Filkins et al. were conducted with co-cultures of $S$. aureus with $P$. aeruginosa, or in the presence of $P$. aeruginosa culture extracts (Filkins et al., 2015), leaving the possibility that other $P$. aeruginosa metabolites contribute to the AQNOdependent shift in $S$. aureus metabolism. Since we showed that HHQ possesses innate antimicrobial activity against $S$. aureus, we assayed gene expression of $S$. aureus treated with HHQ or PQS to determine if either could drive $S$. aureus to fermentative metabolism. While the effects of HHQ were not as robust as that of HQNO, HHQ did cause a small but significant induction of $p f l B$ and $l d h$ expression as compared to the ethanol control (Figure 3B). Moreover, induction of these genes by HHQ was only noted under iron-depleted conditions (Figure 3B-light 


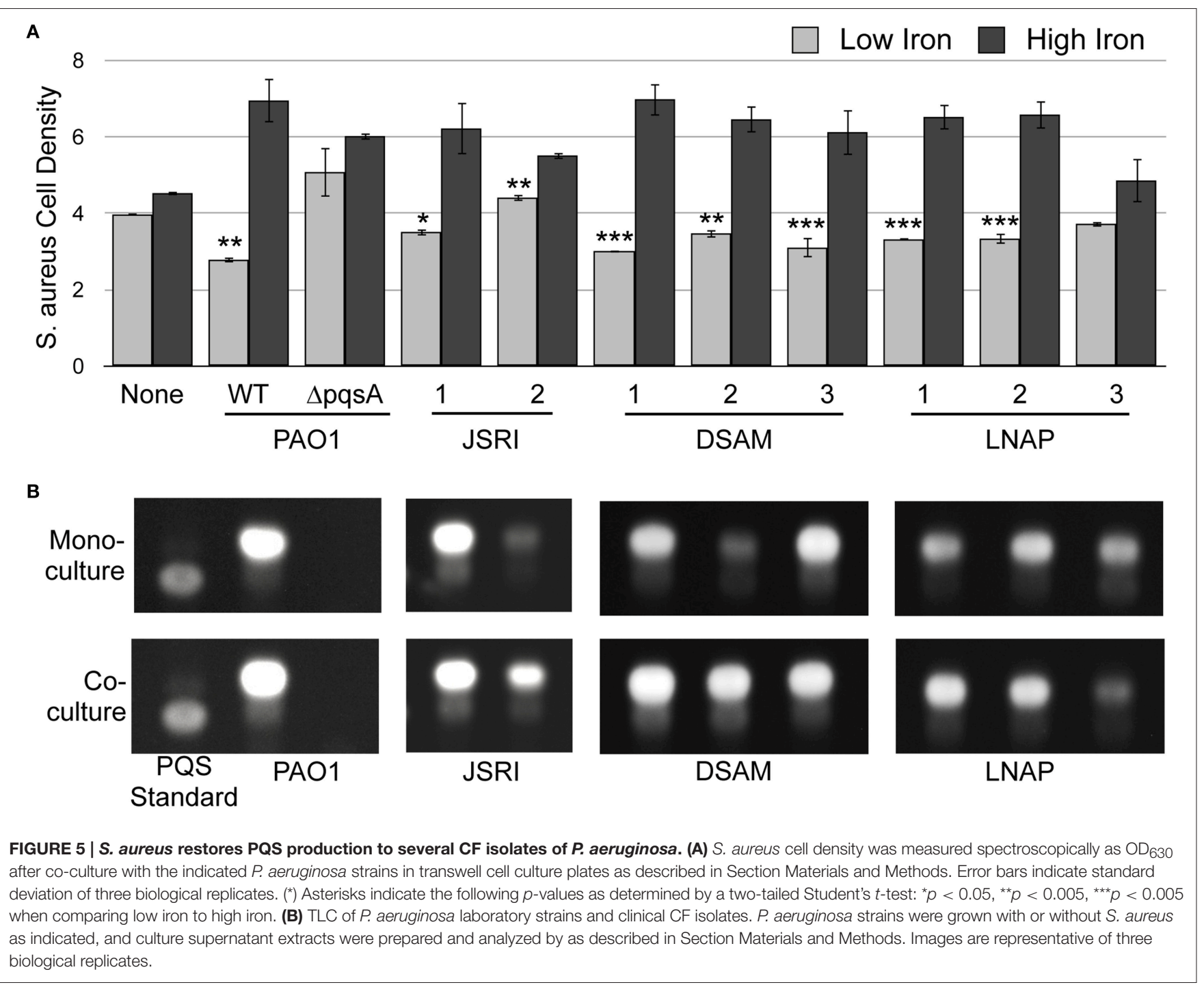

gray bars). In contrast, PQS had no significant effect on $p f l B$, $l d h$, or $a d h$ expression in either high or low iron condition compared to the ethanol control (Figure 3B). Notably, the ability of the individual AQ metabolites to induce fermentative gene expression correlated with their growth suppressive activity against $S$. aureus (Figure 3A). Thus, while HQNO appears to be the major metabolite responsible for increasing expression of $S$. aureus fermentative genes and growth suppressive activity, HHQ is also capable of altering $S$. aureus gene expression related to fermentative metabolism and inhibiting growth of $S$. aureus. Moreover, the sensitivity of $S$. aureus cultures to each of these metabolites is enhanced by iron depletion, presenting a novel means by which iron affects antimicrobial activity of $P$. aeruginosa against $S$. aureus.

\section{Iron-Regulated Antimicrobial Activity is Exhibited by Multiple CF Isolates}

Our previous studies showed that two clonal, longitudinallyisolated strains of $P$. aeruginosa from an individual CF patient exhibited iron-regulated antimicrobial activity against $S$. aureus (Nguyen et al., 2015). To determine if other CF isolates are capable of this activity, we examined clonal, longitudinallyisolated CF strains of $P$. aeruginosa from two additional patients: DSAM (DSAM-1, DSAM-2, and DSAM-3) and LNAP (LNAP1, LNAP-2, and LNAP-3). These strains were isolated from CF patients ranging from age 2 to 22, providing a broad view of $P$. aeruginosa isolates from multiple stages of CF lung infection (Table 1). S. aureus was grown in co-culture with each CF isolate of $P$. aeruginosa, separated by a transwell membrane as previously described (Nguyen et al., 2015), in either high or low iron. With the exception of LNAP-3, each of these isolates exhibited ironregulated antimicrobial activity against $S$. aureus (Figure 5A). As previously shown, the clonal JSRI-1 and JSRI-2 CF isolates also both exhibited iron-regulated antimicrobial activity against $S$. aureus (Figure 5A). These data indicate that iron-regulated antimicrobial activity against $S$. aureus is largely conserved by $P$. aeruginosa throughout CF lung infection, although variations in this activity between individual isolates does exist. 


\section{PQS-Deficient CF Isolates Increase Production of HHQ}

We next determined the potential role of individual AQs produced by CF isolates in iron-regulated antimicrobial activity against $S$. aureus. We previously found that PQS production of the JSRI-2 isolate is reduced as compared to the JSRI1 isolate (Nguyen et al., 2014). However, co-culture with $S$. aureus restores PQS production to JSRI-2, potentially allowing for antimicrobial activity observed by this strain (Nguyen et al., 2015). To determine if PQS production similarly correlates with antimicrobial activity in the LNAP and DSAM CF isolates (Figure 5A), we performed thin layer chromatography (TLC) on culture supernatants from each isolate, grown either in monoculture or co-culture with $S$. aureus. Similar to JSRI-2, DSAM2 showed weak PQS production in monoculture (Figure 5B), yet was still able to exert antimicrobial activity against $S$. aureus (Figure 5A). Also similar to JSRI-2, we found that PQS production of DSAM-2 was restored to this isolate when cocultured with $S$. aureus (Figure 5B). We also noted weak PQS production by LNAP-3 in mono-culture, which was not restored by co-culture with $S$. aureus (Figure 5B). Thus, co-culture with $S$. aureus restores $\mathrm{PQS}$ production to certain $P$. aeruginosa $\mathrm{CF}$ isolates, potentially allowing for iron-regulated antimicrobial activity against $S$. aureus.

Since the data above and our previous work shows that multiple AQs contribute to antimicrobial activity by $P$. aeruginosa laboratory strains (Nguyen et al., 2014), we next examined production of HQNO and HHQ by the JSRI, DSAM, and LNAP isolates. Similar to what was observed for PQS, production of both HQNO and NQNO was reduced in the JSRI2, DSAM-2, and LNAP-3 isolates as compared to clonal isolates from the same patient (Table 2). HHQ and NHQ production were also reduced in JSRI-2 as compared to JSRI-1 (Table 2). In contrast, DSAM-2 and LNAP-3 showed increased production of HHQ and NHQ as compared to clonal isolates from the same patient (Table 2), similar to that observed in the PA14 $\Delta p q s H$ mutant (Figures 4B,C). Combined, these results suggest that JSRI-2 has an overall defect in AQ metabolism, while DSAM2 and LNAP-3 are specifically defective for PQS and AQNO production.

To determine the molecular basis for altered AQ metabolite production by the JSRI-2, DSAM-2, and LNAP-3 isolates, we performed real time PCR of the pqsA and $p q s H$ genes. These analyses show that $p q s A$ gene expression is significantly reduced in the JSRI-2 isolate as compared to JSRI-1, while expression of $p q s H$ is increased in the JSRI-2 isolate (Table 3), supporting the hypothesis that JSRI-2 exhibits an overall defect in AQ metabolism. In contrast, $p q s A$ expression is maintained in DSAM-2 and increased in LNAP-3 as compared to their clonal isolates (Table 3), while $p q s H$ expression is substantially reduced in these strains (Table 3). Thus, defects in the terminal step of PQS synthesis in these isolates correlates with an accumulation of HHQ intermediates. Surprisingly, DSAM-3, which showed no defects in PQS, HHQ, or HQNO production, exhibited significantly reduced expression of both $p q s A$ and $p q s H$ (Table 3). The rationale for this apparent paradox is not clear.
TABLE 2 | Quantification of AQ metabolites in CF isolates.

\begin{tabular}{lcccc}
\hline \multirow{2}{*}{ train } & \multicolumn{4}{c}{ Concentration (nM) } \\
\cline { 2 - 5 } & HQNO & NQNO & HHQ & NHQ \\
\hline JSRI-1 & $2629.7 \pm 580.7$ & $5330.0 \pm 1150.1$ & $80.7 \pm 9.6$ & $285.1 \pm 79.5$ \\
JSRI-2 & $22.8 \pm 7.0^{\star * \star}$ & $3162.4 \pm 1299.6^{\star}$ & $17.5 \pm 8.9^{* \star \star}$ & $26.7 \pm 12.9^{\star \star \star}$ \\
\hline DSAM-1 & $4539.4 \pm 720.6$ & $8342.4 \pm 1657.1$ & $22.7 \pm 2.8$ & $172.2 \pm 27.9$ \\
DSAM-2 & $3073.4 \pm 429.2^{*}$ & $4948.5 \pm 736.2^{*}$ & $903.0 \pm 455.4^{\star \star \star}$ & $515.9 \pm 63.7^{\star \star \star}$ \\
DSAM-3 & $4278.3 \pm 528.2$ & $7957.3 \pm 944.4$ & $25.6 \pm 10.6$ & $275.8 \pm 114.3$ \\
\hline LNAP-1 & $2770.2 \pm 863.4$ & $5699.6 \pm 1718.7$ & $15.6 \pm 6.1$ & $42.5 \pm 16.9$ \\
LNAP-2 & $2996.7 \pm 876.2$ & $5969.8 \pm 1642.0$ & $17.8 \pm 5.5$ & $46.3 \pm 14.0$ \\
LNAP-2 & $1101.0 \pm 280.2^{*}$ & $2098.3 \pm 624.6^{\star \star}$ & $192.4 \pm 83.3^{\star \star}$ & $674.2 \pm 132.8^{\star \star *}$ \\
\hline
\end{tabular}

${ }^{a}$ Determined by LC-MS/MS as described in the Section Materials and Methods. Standard deviation is from five biological replicates. Asterisks (*) indicate the following $p$-values as determined by two-tailed Student's t-test when comparing to the parent strain in each CF series: ${ }^{*} p<0.05,{ }^{* *} p<0.005,{ }^{* \star *} p<0.0005$.

TABLE 3 | pqs gene expression by PQS-deficient CF isolates.

\begin{tabular}{lcc}
\hline Strain & \multicolumn{2}{c}{ Relative expression $^{\mathbf{a}}$ of: } \\
\cline { 2 - 3 } & pqsA & pqsH \\
\hline JSRI-1 & 1.00 & 1.00 \\
JSRI-2 & $0.25 \pm 0.03^{\star \star \star}$ & $3.06 \pm 0.39^{\star \star \star}$ \\
\hline DSAM-1 & 1.00 & 1.00 \\
DSAM-2 & $1.01 \pm 0.24$ & $0.04 \pm 0.02^{\star \star \star}$ \\
DSAM-3 & $0.13 \pm 0.10^{\star \star \star}$ & $0.10 \pm 0.32^{\star \star \star}$ \\
\hline LNAP-1 & 1.00 & 1.00 \\
LNAP-2 & $1.04 \pm 0.37$ & $0.94 \pm 0.24$ \\
LNAP-2 & $5.26 \pm 2.42$ & $0.08 \pm 0.02^{\star \star \star}$
\end{tabular}

${ }^{a}$ Determined by qRT-PCR as described in the Section Materials and Methods. Standard deviation is of three biological replicates. Asterisks $\left(^{*}\right)$ indicate the following p-values as determined by two-tailed Student's t-test when comparing to the parent strain in each CF series: ${ }^{* * *} p<0.0005$.

Overall, these studies suggest that the multifactorial nature of AQ-dependent antimicrobial activity allows $P$. aeruginosa to maintain this activity amid changes in AQ metabolites during CF lung infection.

\section{DISCUSSION}

The decline of $S$. aureus and eventual dominance of $P$. aeruginosa is a common occurrence in the lungs of CF patients (Cystic Fibrosis Foundation, 2014). However, the mechanisms underlying this shift are still not well understood. AQs likely contribute to the ability of $P$. aeruginosa to outcompete $S$. aureus in this environment, as several AQs exhibit growth suppressive properties (Hoffman et al., 2006; Heeb et al., 2011). Furthermore, AQs have been found in the sputum, plasma, and urine of CF patients, highlighting their potential importance in CF infection (Barr et al., 2015). In this study, we show 
that in addition to its roles as a quorum sensing molecule, HHQ possesses innate antimicrobial activity against $S$. aureus. Additionally, we show that $S$. aureus is sensitized to the antimicrobial activity of both HHQ and HQNO when grown under iron limiting environments. Using a combination of LCMS/MS and gene expression analysis, we provide evidence for how CF isolates of $P$. aeruginosa maintain antimicrobial activity during chronic lung infection, despite substantial changes in AQ metabolism. Combined, these results yield many novel insights into how iron affects the progression of $P$. aeruginosa-S. aureus co-infections, demonstrating the complexity of this dynamic microbial interaction.

We previously showed that iron-regulated antimicrobial activity is dependent in part upon AQNOs (Nguyen et al., 2015). Here, we show that iron depletion enhances AQNOdependent antimicrobial activity against $S$. aureus, presenting a novel mechanism by which iron can affect AQ-dependent antimicrobial activity. These are particularly interesting results in light of a recent study from Filkins et al. showing that antimicrobial activity of $P$. aeruginosa against $S$. aureus in mixed biofilms is dependent upon siderophore production, which can similarly reduce extracellular iron levels (Filkins et al., 2015). What remains unknown is the precise mechanism of how AQNOs and iron depletion induce S. aureus to shift toward fermentative metabolism. Respiratory metabolism is heavily dependent upon heme- and iron-cofactored enzymes, and iron starvation has previously been shown to redirect $S$. aureus from respiratory to fermentative metabolism in a manner dependent upon the ferric uptake regulator (Fur; Friedman et al., 2006). Thus, iron depletion may exert an additive effect on $S$. aureus respiratory capabilities in the presence of AQNOs. The implications of this phenomenon for CF disease are particularly interesting, as inhibition of $S$. aureus respiration by HQNO selects for small colony variants (SCVs), which rely on fermentative metabolism and display increased tolerance to multiple antimicrobials (Pan et al., 2002; Proctor et al., 2006, 2014; Lechner et al., 2012; Wood et al., 2013). Notably, the presence of HQNO under our culture conditions reversed the response of $S$. aureus to environmental iron levels (Figure 3A), indicating iron supplementation can help $S$. aureus compensate for the deleterious effects of HQNO on respiratory metabolism. Identifying the specific factors of $S$. aureus that are responsible for regulating this response to HQNO and iron starvation will be critical for understanding the full scope of this microbial interaction.

While iron depletion enhances production of the C9 congener of PQS (Nguyen et al., 2014), the precise contribution of this and related AQ metabolites to overall antimicrobial activity remains elusive. In addition to its role as a quorum signaling molecule, PQS also exhibits iron chelating activity (Bredenbruch et al., 2006; Diggle et al., 2007), which could contribute to antimicrobial activity against $S$. aureus. However, our previous work suggested that PQS is dispensable for this activity, as a $\Delta p q s H$ mutant exhibited antimicrobial activity similar to its wild type parent (Nguyen et al., 2015). While HHQ is not as active as PQS as a quorum signaling molecule (Xiao et al., 2006; Diggle et al., 2007), we noted a substantial increase in HHQ and NHQ production in the $\Delta p q s H$ mutant (Figures $4 \mathbf{B}, \mathbf{C}$ ). The current work further shows that HHQ possesses innate growth suppressive activity against $S$. aureus and can induce expression of $S$. aureus fermentative genes when environmental iron is limiting (Figure 3). While not nearly as active of an antimicrobial as HQNO, these data suggest that HHQ can inhibit $S$. aureus growth by means similar to HQNO, and that overproduction of this metabolite may be able to compensate for $P$. aeruginosa defects in production of PQS and other AQ metabolites.

$P$. aeruginosa undergoes substantial changes as it adapts to the CF lung environment, including the loss of several virulencerelated genes (Smith et al., 2006; Dettman et al., 2013; Huse et al., 2013; Marvig et al., 2014). Our previous studies showed that PQS production was reduced in at least one CF isolate (JSRI-2) over time in the CF lung (Nguyen et al., 2014). Despite this reduction, the JSRI-2 isolate retained the ability to mediate iron-regulated antimicrobial activity, potentially due to the finding that coculture with $S$. aureus restored PQS production to this isolate (Nguyen et al., 2015). In the current study, analysis of clonal and longitudinal isolates from additional CF patients has provided further models into how $P$. aeruginosa retains antimicrobial activity during $\mathrm{CF}$ lung infection. Similar to the isogenic $\Delta p q s H$ mutant, overproduction of HHQ by CF isolates has the potential to compensate for loss of PQS production in these isolates, and thus allow $P$. aeruginosa to retain antimicrobial activity against $S$. aureus.

It is important to note that the isolates analyzed in this study represent only a small subset of the $P$. aeruginosa community in the CF lung, which has been shown to be highly heterogeneous (Dettman et al., 2013; Winstanley et al., 2016). Thus, it is possible that AQ metabolites produced by other members of the community allowed for the emergence of metabolic cheats, as has been noted in multiple studies (Andersen et al., 2015; RossGillespie et al., 2015). The finding that nearly all of the CF isolates we have thus far tested exhibit iron-regulated antimicrobial activity against $S$. aureus points to this phenomenon being independent of the cheater paradigm, at least in the context of mixed $P$. aeruginosa communities. Instead, it is likely that the multifactorial nature of iron-regulated antimicrobial activity allows it to persist in spite of changes incurred to AQ metabolites. It is also important to note that, with the exception of the transwell co-culture assays, the experiments in this study were performed on shaking, planktonic cultures, while microbial growth in the CF lung occurs in biofilms. It is possible that the antimicrobial activity of individual AQs against $S$. aureus biofilms could differ from that of planktonic cells to some extent. However, previous work by Filkins et al. has shown that both AQ production and iron availability contribute to $P$. aeruginosa's antimicrobial activity against $S$. aureus in biofilms. Thus, the results that we show here are likely translatable to mixed biofilm growth of these two species.

Iron is an essential nutrient for both $P$. aeruginosa and $S$. aureus and is increasingly appreciated as a critical mediator of CF lung disease (Cassat and Skaar, 2013; Barnabie and Whiteley, 2015; Bouvier, 2016). The finding that iron modulates antimicrobial activity of multiple $P$. aeruginosa CF isolates further indicates the importance of this essential nutrient in 
progression of CF lung disease. While siderophores are critical for antimicrobial activity in laboratory strains of $P$. aeruginosa (Filkins et al., 2015), siderophore-mediated iron uptake is less likely to be important for this process in the CF lung due to reduced dependence on these systems as disease progresses (Marvig et al., 2014; Nguyen et al., 2014). Determining how primary clinical isolates of $P$. aeruginosa mediate antimicrobial activity in the context of CF lung infection will therefore require further studies of iron uptake and regulation by primary CF isolates, and well as determining the impact of relevant host immune factors that sequester host iron sources. Most importantly, this work demonstrates the importance of considering iron and other essential nutrients when studying microbial interactions in the context of human disease.

\section{AUTHOR CONTRIBUTIONS}

AN, JJ, MC, PW, MK, AO contributed substantially to the conception/design, acquisition, and analysis of this work. AN, JJ, $\mathrm{MC}, \mathrm{PW}, \mathrm{MK}, \mathrm{AO}$ revised the work for intellectual content and

\section{REFERENCES}

Andersen, S. B., Marvig, R. L., Molin, S., Krogh Johansen, H., and Griffin, A. S. (2015). Long-term social dynamics drive loss of function in pathogenic bacteria. Proc. Natl. Acad. Sci. U.S.A. 112, 10756-10761. doi: 10.1073/pnas.1508324112

Barnabie, P. M., and Whiteley, M. (2015). Iron-mediated control of Pseudomonas aeruginosa-Staphylococcus aureus interactions in the cystic fibrosis lung. J. Bacteriol. 197, 2250-2251. doi: 10.1128/JB.00303-15

Barr, H. L., Halliday, N., Camara, M., Barrett, D. A., Williams, P., Forrester, D. L., et al. (2015). Pseudomonas aeruginosa quorum sensing molecules correlate with clinical status in cystic fibrosis. Eur. Respir. J. 46, 1046-1054. doi: $10.1183 / 09031936.00225214$

Bhatt, J. M. (2013). Treatment of pulmonary exacerbations in cystic fibrosis. Eur. Respir. Rev. 22, 205-216. doi: 10.1183/09059180.00006512

Bouvier, N. M. (2016). Cystic fibrosis and the war for iron at the hostpathogen battlefront. Proc. Natl. Acad. Sci. U.S.A. 113, 1480-1482. doi: 10.1073/pnas.1525101113

Bredenbruch, F., Geffers, R., Nimtz, M., Buer, J., and Haussler, S. (2006). The Pseudomonas aeruginosa quinolone signal (PQS) has an iron-chelating activity. Environ. Microbiol. 8, 1318-1329. doi: 10.1111/j.1462-2920.2006.01025.x

Calfee, M. W., Coleman, J. P., and Pesci, E. C. (2001). Interference with Pseudomonas quinolone signal synthesis inhibits virulence factor expression by Pseudomonas aeruginosa. Proc. Natl. Acad. Sci. U.S.A. 98, 11633-11637. doi: 10.1073/pnas.201328498

Cassat, J. E., and Skaar, E. P. (2013). Iron in infection and immunity. Cell Host Microbe. 13, 509-519. doi: 10.1016/j.chom.2013.04.010

Coleman, J. P., Hudson, L. L., McKnight, S. L., Farrow, J. M. III, Calfee, M. W., Lindsey, C. A., et al. (2008). Pseudomonas aeruginosa PqsA is an anthranilatecoenzyme A ligase. J. Bacteriol. 190, 1247-1255. doi: 10.1128/JB.01140-07

Collier, D. N., Anderson, L., McKnight, S. L., Noah, T. L., Knowles, M., Boucher, R., et al. (2002). A bacterial cell to cell signal in the lungs of cystic fibrosis patients. FEMS Microbiol. Lett. 215, 41-46. doi: 10.1111/j.1574-6968.2002.tb11367.x

Cornelis, P. (2010). Iron uptake and metabolism in pseudomonads. Appl. Microbiol. Biotechnol. 86, 1637-1645. doi: 10.1007/s00253-010-2550-2

Cornelis, P., and Dingemans, J. (2013). Pseudomonas aeruginosa adapts its iron uptake strategies in function of the type of infections. Front. Cell. Infect. Microbiol. 3:75. doi: 10.3389/fcimb.2013.00075

D’Argenio, D. A., Calfee, M. W., Rainey, P. B., and Pesci, E. C. (2002). Autolysis and autoaggregation in Pseudomonas aeruginosa colony morphology mutants. J. Bacteriol. 184, 6481-6489. doi: 10.1128/JB.184.23.6481-6489.2002

Dettman, J. R., Rodrigue, N., Aaron, S. D., and Kassen, R. (2013). Evolutionary genomics of epidemic and nonepidemic strains of Pseudomonas aeruginosa. approved the final version for publication. AN, JJ, MC, PW, MK, $\mathrm{AO}$ agree to be accountable for all aspects of this work.

\section{ACKNOWLEDGMENTS}

This work was supported by NIH-NIAID Contract HHSN272201000046C (to MK), the University of Maryland School of Pharmacy Mass Spectrometry Center (SOP1841IQB2014-to MK), start-up funds from the University of Maryland School of Pharmacy (to AO and MK), the Biological and Biotechnological Sciences Research Council U.K. (BBF0143921), the JPI-AMR-Medical Research Council (MR/N501852/1; to MC and PW), and University of Maryland, Baltimore County CBI Training Grant T32 GM 066706 (to AN).

\section{SUPPLEMENTARY MATERIAL}

The Supplementary Material for this article can be found online at: http://journal.frontiersin.org/article/10.3389/fmicb. 2016.01171

Proc. Natl. Acad. Sci. U.S.A. 110, 21065-21070. doi: 10.1073/pnas.13078 62110

Deziel, E., Lepine, F., Milot, S., He, J., Mindrinos, M. N., Tompkins, R. G., et al. (2004). Analysis of Pseudomonas aeruginosa 4-hydroxy-2alkylquinolines (HAQs) reveals a role for 4-hydroxy-2-heptylquinoline in cellto-cell communication. Proc. Natl. Acad. Sci. U.S.A. 101, 1339-1344. doi: 10.1073/pnas.0307694100

Dietrich, L. E., Price-Whelan, A., Petersen, A., Whiteley, M., and Newman, D. K. (2006). The phenazine pyocyanin is a terminal signalling factor in the quorum sensing network of Pseudomonas aeruginosa. Mol. Microbiol. 61, 1308-1321. doi: 10.1111/j.1365-2958.2006.05306.x

Diggle, S. P., Matthijs, S., Wright, V. J., Fletcher, M. P., Chhabra, S. R., Lamont, I. L., et al. (2007). The Pseudomonas aeruginosa 4-quinolone signal molecules HHQ and PQS play multifunctional roles in quorum sensing and iron entrapment. Chem. Biol. 14, 87-96. doi: 10.1016/j.chembiol.2006.11.014

Drees, S. L., Li, C., Prasetya, F., Saleem, M., Dreveny, I., Williams, P., et al. (2016). PqsBC, a condensing enzyme in the biosynthesis of the Pseudomonas aeruginosa quinolone signal: crystal structure, inhibition, and reaction mechanism. J. Biol. Chem. 291, 6610-6624. doi: 10.1074/jbc.M115.708453

Dulcey, C. E., Dekimpe, V., Fauvelle, D. A., Milot, S., Groleau, M. C., Doucet, N., et al. (2013). The end of an old hypothesis: the pseudomonas signaling molecules 4-hydroxy-2-alkylquinolines derive from fatty acids, not 3-ketofatty acids. Chem. Biol. 20, 1481-1491. doi: 10.1016/j.chembiol.2013.09.021

Filkins, L. M., Graber, J. A., Olson, D. G., Dolben, E. L., Lynd, L. R., Bhuju, S., et al. (2015). Co-culture of Staphylococcus aureus with Pseudomonas aeruginosa drives $S$. aureus towards fermentative metabolism and reduced viability in a cystic fibrosis model. J. Bacteriol. 197, 2252-2264. doi: 10.1128/JB.00059-15

Cystic Fibrosis Foundation (2014). Patient Registry Annual Data Report 2014. Bethesda, MLD: Cystic Fibrosis Foundation.

Friedman, D. B., Stauff, D. L., Pishchany, G., Whitwell, C. W., Torres, V. J., and Skaar, E. P. (2006). Staphylococcus aureus redirects central metabolism to increase iron availability. PLoS Pathog. 2:e87. doi: 10.1371/journal.ppat.0020087

Harro, J. M., Daugherty, S., Bruno, V. M., Jabra-Rizk, M. A., Rasko, D. A., and Shirtliff, M. E. (2013). Draft genome sequence of the methicillin-resistant Staphylococcus aureus isolate MRSA-M2. Genome Announc. 1:e00037-12. doi: 10.1128/genomeA.00037-12

Heeb, S., Fletcher, M. P., Chhabra, S. R., Diggle, S. P., Williams, P., and Camara, M. (2011). Quinolones: from antibiotics to autoinducers. FEMS Microbiol. Rev. 35, 247-274. doi: 10.1111/j.1574-6976.2010.00247.x

Hoffman, L. R., Deziel, E., D’Argenio, D. A., Lepine, F., Emerson, J., McNamara, S., et al. (2006). Selection for Staphylococcus aureus small-colony variants due to 
growth in the presence of Pseudomonas aeruginosa. Proc. Natl. Acad. Sci. U.S.A. 103, 19890-19895. doi: 10.1073/pnas.0606756104

Hoiby, N., Ciofu, O., and Bjarnsholt, T. (2010). Pseudomonas aeruginosa biofilms in cystic fibrosis. Future Microbiol. 5, 1663-1674. doi: 10.2217/fmb. 10.125

Holloway, B. W. (1955). Genetic recombination in Pseudomonas aeruginosa. J. Gen. Microbiol. 13, 572-581. doi: 10.1099/00221287-13-3-572

Huse, H. K., Kwon, T., Zlosnik, J. E., Speert, D. P., Marcotte, E. M., and Whiteley, M. (2013). Pseudomonas aeruginosa enhances production of a non-alginate exopolysaccharide during long-term colonization of the cystic fibrosis lung. PLoS ONE 8:e82621. doi: 10.1371/journal.pone.0082621

Ilangovan, A., Fletcher, M., Rampioni, G., Pustelny, C., Rumbaugh, K., Heeb, S., et al. (2013). Structural basis for native agonist and synthetic inhibitor recognition by the Pseudomonas aeruginosa quorum sensing regulator PqsR (MvfR). PLoS Pathog. 9:e1003508. doi: 10.1371/journal.ppat.10 03508

Kessler, E., Safrin, M., Abrams, W. R., Rosenbloom, J., and Ohman, D. E. (1997). Inhibitors and specificity of Pseudomonas aeruginosa LasA. J. Biol. Chem. 272, 9884-9889. doi: 10.1074/jbc.272.15.9884

Kessler, E., Safrin, M., Olson, J. C., and Ohman, D. E. (1993). Secreted LasA of Pseudomonas aeruginosa is a staphylolytic protease. J. Biol. Chem. 268, 7503-7508.

Konings, A. F., Martin, L. W., Sharples, K. J., Roddam, L. F., Latham, R., Reid, D. W., et al. (2013). Pseudomonas aeruginosa uses multiple pathways to acquire iron during chronic infection in cystic fibrosis lungs. Infect. Immun. 81, 2697-2704. doi: 10.1128/IAI.00418-13

Korgaonkar, A., Trivedi, U., Rumbaugh, K. P., and Whiteley, M. (2013). Community surveillance enhances Pseudomonas aeruginosa virulence during polymicrobial infection. Proc. Natl. Acad. Sci. U.S.A. 110, 1059-1064. doi: 10.1073/pnas. 1214550110

Lechner, S., Lewis, K., and Bertram, R. (2012). Staphylococcus aureus persisters tolerant to bactericidal antibiotics. J. Mol. Microbiol. Biotechnol. 22, 235-244. doi: $10.1159 / 000342449$

Lightbown, J. W., and Jackson, F. L. (1956). Inhibition of cytochrome systems of heart muscle and certain bacteria by the antagonists of dihydrostreptomycin: 2-alkyl-4-hydroxyquinoline N-oxides. Biochem. J. 63, 130-137. doi: 10.1042/bj0630130

Machan, Z. A., Taylor, G. W., Pitt, T. L., Cole, P. J., and Wilson, R. (1992). 2-Heptyl-4-hydroxyquinoline $\mathrm{N}$-oxide, an antistaphylococcal agent produced by Pseudomonas aeruginosa. J. Antimicrob. Chemother. 30, 615-623. doi: $10.1093 / \mathrm{jac} / 30.5 .615$

Marvig, R. L., Damkiaer, S., Khademi, S. M., Markussen, T. M., Molin, S., and Jelsbak, L. (2014). Within-host evolution of Pseudomonas aeruginosa reveals adaptation toward iron acquisition from hemoglobin. mBio 5, e00966-14. doi: 10.1128/mBio.00966-14

Mashburn, L. M., Jett, A. M., Akins, D. R., and Whiteley, M. (2005). Staphylococcus aureus serves as an iron source for Pseudomonas aeruginosa during in vivo coculture. J. Bacteriol. 187, 554-566. doi: 10.1128/JB.187.2.554-56 6.2005

Meyer, J. M., Neely, A., Stintzi, A., Georges, C., and Holder, I. A. (1996). Pyoverdine is essential for virulence of Pseudomonas aeruginosa. Infect. Immun. $64,518-523$.

Nadal Jimenez, P., Koch, G., Papaioannou, E., Wahjudi, M., Krzeslak, J., Coenye, T., et al. (2010). Role of PvdQ in Pseudomonas aeruginosa virulence under iron-limiting conditions. Microbiology 156(Pt 1), 49-59. doi: 10.1099/mic.0.03 0973-0

Nairz, M., Schroll, A., Sonnweber, T., and Weiss, G. (2010). The struggle for iron - a metal at the host-pathogen interface. Cell. Microbiol. 12, 1691-1702. doi: 10.1111/j.1462-5822.2010.01529.x

Nguyen, A. T., Jones, J. W., Ruge, M. A., Kane, M. A., and Oglesby-Sherrouse, A. G. (2015). Iron depletion enhances production of antimicrobials by Pseudomonas aeruginosa. J. Bacteriol. 197, 2265-2275. doi: 10.1128/JB.00072-15

Nguyen, A. T., O’Neill, M. J., Watts, A. M., Robson, C. L., Lamont, I. L., Wilks, A., et al. (2014). Adaptation of iron homeostasis pathways by a Pseudomonas aeruginosa pyoverdine mutant in the cystic fibrosis lung. J. Bacteriol. 196, 2265-2276. doi: 10.1128/JB.01491-14

Oglesby, A. G., Farrow, J. M. III, Lee, J. H., Tomaras, A. P., Greenberg, E. P., Pesci, E. C., et al. (2008). The influence of iron on Pseudomonas aeruginosa physiology: a regulatory link between iron and quorum sensing. J. Biol. Chem. 283, 15558-15567. doi: 10.1074/jbc.M707840200

Oglesby-Sherrouse, A. G., Djapgne, L., Nguyen, A. T., Vasil, A. I., and Vasil, M. L. (2014). The complex interplay of iron, biofilm formation, and mucoidy affecting antimicrobial resistance of Pseudomonas aeruginosa. Pathog. Dis. 70, 307-320. doi: 10.1111/2049-632X.12132

Ortori, C. A., Dubern, J. F., Chhabra, S. R., Camara, M., Hardie, K., Williams, P., et al. (2011). Simultaneous quantitative profiling of N-acyl-L-homoserine lactone and 2-alkyl-4(1H)-quinolone families of quorum-sensing signaling molecules using LC-MS/MS. Anal. Bioanal. Chem. 399, 839-850. doi: 10.1007/s00216-010-4341-0

Otto, B. R., Verweij-van Vught, A. M., and MacLaren, D. M. (1992). Transferrins and heme-compounds as iron sources for pathogenic bacteria. Crit. Rev. Microbiol. 18, 217-233. doi: 10.3109/10408419209114559

Pan, X. S., Hamlyn, P. J., Talens-Visconti, R., Alovero, F. L., Manzo, R. H., and Fisher, L. M. (2002). Small-colony mutants of Staphylococcus aureus allow selection of gyrase-mediated resistance to dual-target fluoroquinolones. Antimicrob. Agents Chemother. 46, 2498-2506. doi: 10.1128/AAC.46.8.24982506.2002

Pesci, E. C., Milbank, J. B., Pearson, J. P., McKnight, S., Kende, A. S., Greenberg, E. P., et al. (1999). Quinolone signaling in the cell-to-cell communication system of Pseudomonas aeruginosa. Proc. Natl. Acad. Sci. U.S.A. 96, 11229-11234. doi: 10.1073/pnas.96.20.11229

Price-Whelan, A., Dietrich, L. E., and Newman, D. K. (2006). Rethinking 'secondary' metabolism: physiological roles for phenazine antibiotics. Nat. Chem. Biol. 2, 71-78. doi: 10.1038/nchembio764

Proctor, R. A., Kriegeskorte, A., Kahl, B. C., Becker, K., Loffler, B., and Peters, G. (2014). Staphylococcus aureus Small Colony Variants (SCVs): a road map for the metabolic pathways involved in persistent infections. Front. Cell. Infect. Microbiol. 4:99. doi: 10.3389/fcimb.2014.00099

Proctor, R. A., von Eiff, C., Kahl, B. C., Becker, K., McNamara, P., Herrmann, M., et al. (2006). Small colony variants: a pathogenic form of bacteria that facilitates persistent and recurrent infections. Nat. Rev. Microbiol. 4, 295-305. doi: $10.1038 /$ nrmicro 1384

Rabin, H. R., Butler, S. M., Wohl, M. E., Geller, D. E., Colin, A. A., Schidlow, D. V., et al. (2004). Pulmonary exacerbations in cystic fibrosis. Pediatr. Pulmonol. 37, 400-406. doi: 10.1002/ppul.20023

Rahme, L. G., Stevens, E. J., Wolfort, S. F., Shao, J., Tompkins, R. G., and Ausubel, F. M. (1995). Common virulence factors for bacterial pathogenicity in plants and animals. Science 268, 1899-1902. doi: 10.1126/science.7604262

Rampioni, G., Pustelny, C., Fletcher, M. P., Wright, V. J., Bruce, M., Rumbaugh, K. P., et al. (2010). Transcriptomic analysis reveals a global alkyl-quinoloneindependent regulatory role for PqsE in facilitating the environmental adaptation of Pseudomonas aeruginosa to plant and animal hosts. Environ. Microbiol. 12, 1659-1673. doi: 10.1111/j.1462-2920.2010.02214.x

Reinhart, A. A., Powell, D. A., Nguyen, A. T., O’Neill, M., Djapgne, L., Wilks, A., et al. (2015). The prrF-encoded small regulatory RNAs are required for iron homeostasis and virulence of Pseudomonas aeruginosa. Infect. Immun. 83, 863-875. doi: 10.1128/IAI.02707-14

Ross-Gillespie, A., Dumas, Z., and Kummerli, R. (2015). Evolutionary dynamics of interlinked public goods traits: an experimental study of siderophore production in Pseudomonas aeruginosa. J. Evol. Biol. 28, 29-39. doi: 10.1111/jeb.12559

Rothery, R. A., and Weiner, J. H. (1996). Interaction of an engineered [3Fe-4S] cluster with a menaquinol binding site of Escherichia coli DMSO reductase. Biochemistry 35, 3247-3257. doi: 10.1021/bi951584y

Smirnova, I. A., Hagerhall, C., Konstantinov, A. A., and Hederstedt, L. (1995). HOQNO interaction with cytochrome b in succinate:menaquinone oxidoreductase from Bacillus subtilis. FEBS Lett. 359, 23-26. doi: 10.1016/00145793(94)01442-4

Smith, E. E., Buckley, D. G., Wu, Z., Saenphimmachak, C., Hoffman, L. R., D’Argenio, D. A., et al. (2006). Genetic adaptation by Pseudomonas aeruginosa to the airways of cystic fibrosis patients. Proc. Natl. Acad. Sci. U.S.A. 103, 8487-8492. doi: 10.1073/pnas.0602138103

Takase, H., Nitanai, H., Hoshino, K., and Otani, T. (2000). Impact of siderophore production on Pseudomonas aeruginosa infections in immunosuppressed mice. Infect. Immun. 68, 1834-1839. doi: 10.1128/IAI.68.4.1834-183 9.2000 
Van Ark, G., and Berden, J. A. (1977). Binding of HQNO to beef-heart sub-mitochondrial particles. Biochim. Biophys. Acta 459, 119-127. doi: 10.1016/0005-2728(77)90014-7

Winstanley, C., O’Brien, S., and Brockhurst, M. A. (2016). Pseudomonas aeruginosa evolutionary adaptation and diversification in cystic fibrosis chronic lung infections. Trends Microbiol. 24, 327-337. doi: 10.1016/j.tim.2016.01.008

Wood, T. K., Knabel, S. J., and Kwan, B. W. (2013). Bacterial persister cell formation and dormancy. Appl. Environ. Microbiol. 79, 7116-7121. doi: 10.1128/AEM.02636-13

Wratten, S. J., Wolfe, M. S., Andersen, R. J., and Faulkner, D. J. (1977). Antibiotic metabolites from a marine pseudomonad. Antimicrob. Agents Chemother. 11, 411-414. doi: 10.1128/AAC.11.3.411

Xiao, G., Deziel, E., He, J., Lepine, F., Lesic, B., Castonguay, M. H., et al. (2006). MvfR, a key Pseudomonas aeruginosa pathogenicity LTTR-class regulatory protein, has dual ligands. Mol. Microbiol. 62, 1689-1699. doi: 10.1111/j.13652958.2006.05462.x
Xiong, Y. Q., Vasil, M. L., Johnson, Z., Ochsner, U. A., and Bayer, A. S. (2000). The oxygen- and iron-dependent sigma factor $p v d S$ of Pseudomonas aeruginosa is an important virulence factor in experimental infective endocarditis. J. Infect. Dis. 181, 1020-1026. doi: 10.1086/315338

Conflict of Interest Statement: The authors declare that the research was conducted in the absence of any commercial or financial relationships that could be construed as a potential conflict of interest.

Copyright (C) 2016 Nguyen, Jones, Cámara, Williams, Kane and Oglesby-Sherrouse. This is an open-access article distributed under the terms of the Creative Commons Attribution License (CC BY). The use, distribution or reproduction in other forums is permitted, provided the original author(s) or licensor are credited and that the original publication in this journal is cited, in accordance with accepted academic practice. No use, distribution or reproduction is permitted which does not comply with these terms. 